\title{
Active control of the wake behind the cylinder
}

\author{
Zuzana Broučková $^{1}$, Erik Flídr ${ }^{1,3}$, Pavel Šafařík ${ }^{2}$ and Zdeněk Trávníček ${ }^{1}$ \\ ${ }^{1}$ Institute of Thermomechanics, The Czech Academy of Sciences, Prague, Czech Republic \\ ${ }^{2}$ Czech Technical University, Faculty of Mechanical Engineering, 16607 Prague, Czech Republic \\ ${ }^{3}$ VZLÚ, Beranových 130, 19905 Prague - Letňany
}

\begin{abstract}
Active control of the flow around a circular cylinder using a synthetic jet (SJ) array was experimentally investigated in a small wind tunnel. The prevailing method was flow visualization. Modification of the wake was investigated at two values of a dimensionless momentum coefficient $\left(C_{\mu}=0.21\right.$ and 1.02). Various $\alpha$ and $C_{\mathrm{f}}$ parameters were tested (where $\alpha$ is the angle between SJs and the main flow direction and $C_{\mathrm{f}}$ is the frequency ratio). It was found that the control moves a separation point more downstream, i.e. the width of the wake can be reduced. Moreover, increasing frequency ratio $C_{\mathrm{f}}$ causes refining of the vortex structure. When the SJs are placed at the front or rear stagnation points, the wake tends towards the symmetrical pattern.
\end{abstract}

\section{Introduction}

Based on a need of energy input flow control methods can be categorized into passive and active [1]. Synthetic jet (SJ) is one of the active methods used for flow control. Formation and evaluation of the SJ was investigated in [2, 3]. An influence of the active flow control using SJs on the flow around a circular cylinder was extensively studied by many researches. Some important findings concerning these cases were summarized in [4], more detailed view on the topic can be found in [5].

In [6] was shown if the angle between the SJ and oncoming flow $\alpha$ (see Figure 1) and the dimensionless momentum coefficient $C_{\mu}$ (see Eq.(4) in the text below) were optimal, then the drag coefficient was reduced about $30 \%$. If the SJ was placed in the rear stagnation point, small close envelope was formed during the extrusion part of the period [7]. During the intrusion part of the period the vortex pair is convected downstream from the both sides of the cylinder and interacts with the wake. In [8] it was found that vortex shedding under the flow control can be categorized into six regimes.

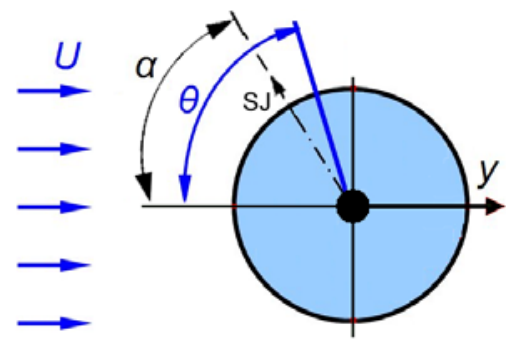

Fig. 1. Coordinate system of the cylinder in cross flow.

All the above mentioned investigations used harmonic driving signal (sinusoidal wave). Another approach was tested in $[9,10]$. If the extrusion and intrusion part of period is changed (typically, the duty cycle is changed), the $C_{\mu}$ coefficient is changed too without frequency changing. With increasing frequency of synthetic jet, the lock-on effect was observed in [11, $18,19]$. In [12] two roughness strips (i.e. passive control) were used in combination with synthetic jet (i.e. active control).

In this paper, an influence of the angle $\alpha$, SJ frequency and coefficient $C_{\mu}$ was studied by means of flow visualization.

\section{Parameters}

For description of the flow around a cylinder two dimensionless numbers were introduced in [13]. Based on the oncoming flow velocity $U$ and the cylinder diameter $d$ the Reynolds number is defined as $R e=U \cdot d / v$, where $v$ is the kinematic viscosity The Strouhal number is defined as $S t=f \cdot d / U$, where $f$ is the natural vortex shedding frequency.

For SJ the Reynolds number is defined as $R e_{\mathrm{U}_{0}}=U_{0} D_{\mathrm{o}} / v$, where $U_{0}$ is time-mean velocity based on the extrusion part of the period and $D_{\mathrm{o}}$ is the diameter of the actuator output,

$$
U_{0}=\frac{1}{T} \int_{0}^{T / 2} u_{0}(t) d t,
$$

where $u_{0}(t)$ is the SJ exit velocity in time $(t)$, and $T$ is the period.

Strouhal number of the SJ is defined as $S t_{\mathrm{U}_{0}}=f_{\mathrm{sj}} D_{\mathrm{o}} / U_{0}$, where $f_{\mathrm{sj}}=1 / T$ is the $\mathrm{SJ}$ frequency. The jet momentum is generated during the extrusion part of the actuating period. Assuming the slug flow model

* Corresponding author: brouckova@it.cas.cz

(C) The Authors, published by EDP Sciences. This is an open access article distributed under the terms of the Creative Commons Attribution License 4.0 (http://creativecommons.org/licenses/by/4.0/). 
and the sinusoidal waveform of the actuation signal, in general, the jet momentum can be calculated as

$$
M_{s j}=\frac{1}{T} \int_{0}^{T / 2} \rho A u^{2}(t) d t,
$$

where $\rho$ is the fluid density, $A$ is the area of the actuator output.

For describing of the flow control it is appropriate to defined some dimensionless ratios. The frequency ratio is defined as

$$
C_{\mathrm{f}}=\frac{f_{\mathrm{sj}}}{f} .
$$

The momentum coefficient is defined in [6] as

$$
C_{\mu}=\frac{M_{\mathrm{sj}}}{M}
$$

where $M$ is the oncoming flow momentum.

\section{Experimental setup and methods}

\subsection{Synthetic jet actuator}

Figure 2 shows a schematic view of the SJ actuator including a coordinate system. The actuator consisted of a cavity with a height of $10 \mathrm{~mm}$, which was equipped with an electrodynamically driven diaphragm. Diaphragm originated from loudspeaker Monacor SP$7 / 4 \mathrm{~S}$, powered by function generator FG7002C. The diaphragm was characterized by its diameter $D_{\mathrm{D}}=53 \mathrm{~mm}$ and rigidity $K_{\mathrm{p}}=4.8 \cdot 10^{5} \mathrm{~N} \cdot \mathrm{m}^{-3}[14]$. The cavity was connected with the surroundings by the pipe with the inner diameter $D=3 \mathrm{~mm}$, the outer diameter $d=4 \mathrm{~mm}$ and the length $L=54 \mathrm{~mm}$. End of the pipe was placed into the flow. The pipe was placed perpendicularly to the main flow, its end part touched the front wall of the tunnel. In the part of the pipe which was submerged in the main flow $(l=29 \mathrm{~mm}) 14$ actuator outputs with diameter $D_{\mathrm{o}}=1.05 \mathrm{~mm}$ were placed in one row with distance of $2 \mathrm{~mm}$ (see Figure 2).

For the particular case of the present actuator, the momentum coefficient, defined by Eq. (4), was evaluated in [20] as

$$
C_{\mu}=\frac{7 \pi^{3}}{8} \frac{D_{o}^{2}}{d l}\left(\frac{U_{0}}{U}\right)^{2} .
$$

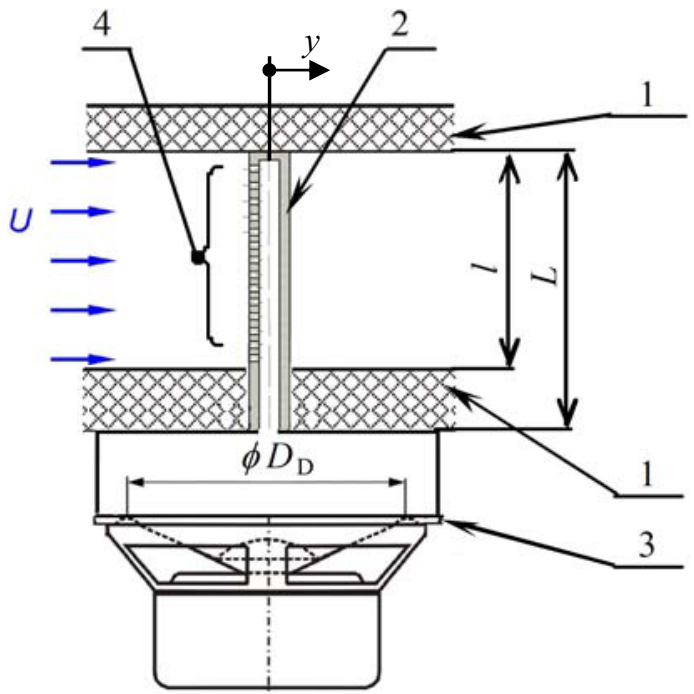

Fig. 2. Top-view scheme of the cylinder in the wind tunnel: 1: tunnel side walls, 2: cylinder (outer diameter of $d=4 \mathrm{~mm}$ ), 3: SJ actuator, 4: row of 14 SJs (diameters of $D_{\mathrm{o}}=1.05 \mathrm{~mm}$, equidistantly distributed with spacing of $2 \mathrm{~mm}$ ).

\subsection{Flow visualization equipment}

Flow visualization was performed in a small wind tunnel with a test section $(460 \times 350 \times 29) \mathrm{mm}=($ length $\times$ height $\times$ depth). The free stream velocity was $U=0.77$ $\mathrm{m} \cdot \mathrm{s}^{-1}$, i.e. the corresponding Reynolds number was $R e=193$. Visualization medium was water fog created by the ultrasonic generator MiniNebler. During the experiments continual or stroboscopic light was used for illumination of the test section.

The light system for the continual or stroboscopic regime is an inhouse-built system based on the battery of 10 LEDs (Hi-Power HPA8B-49k5WHC, 5W of each) designed for the operation frequency of $0-500 \mathrm{~Hz}$ and the light pulse duration of $75-3500 \mu \mathrm{s}$. The system enables to synchronize the stroboscopic light with the driven frequency at an arbitrary phase of the driven cycle.

Pictures were taken by digital camera Canon PowerShot G7 connected to a PC via USB cable, using exposures of 1s. The photos were multi-exposures of a large number of frames (in range from 27 to 162 frames).

\subsection{Pitot tube}

The time mean velocity was measured by the Pitot tube (blunt-nosed impact tube, with the outer and inner diameter of $2 \mathrm{~mm}$ and $1.5 \mathrm{~mm}$, respectively), connected to an electronic manometer GMH 3156 with GMSD 2.5 transducer with resolution of $0.1 \mathrm{~Pa}$. From Bernoulli's equation, the flow velocity can be calculated as $u=c_{\mathrm{p}}\left[2\left(p-p_{\mathrm{s}}\right) / \rho\right]^{0.5}$ where $p$ is the total pressure, $p_{\mathrm{s}}$ is the static pressure (the static pressure was considered equal to the barometric pressure) and $c_{\mathrm{p}}$ is the pressure coefficient (approximately 1.0 in agreement with the Bernoulli's equation). 
In the present study, the Pitot tube was used for measurement of frequency and power characteristic. In [14] was shown that the Pitot tube placed far enough from the actuator output can be used for pulsatile flow measurement. Therefore, the $\mathrm{SJ}$ time-mean velocity, $U_{0}$, was estimated from the Pitot tube measurement (similarly as in [14]).

\section{Results and discussion}

\subsection{Frequency and power characteristics}

In order to keep the momentum coefficient $C_{\mu}$ constant, the power of the SJ actuator was varied to compensate velocity changes caused by changes of the frequency. For that reason, the frequency and power characteristics were measured.

The Pitot tube was placed at $y / D_{0}=6$ and $z=0 \mathrm{~mm}$. The measurement points were distributed equidistantly along the $x$-axis, with the spacing $2 \mathrm{~mm}$. The first point was placed at $x=14 \mathrm{~mm}$ and the last one at $x=40 \mathrm{~mm}$. The frequency characteristic was evaluated over the range of $f=30-150 \mathrm{~Hz}$. During the measurement, an input power of the SJ actuator was set as the constant of $P=2 \mathrm{~W}$. It vas evaluated as the product of the measured supply current and voltage (both effective values; the phase angle between them was neglected similarly as in [14]).

Figure 3 shows the frequency characteristic evaluated experimentally. Moreover, the theoretical resonance frequency is shown too (for derivation details see [20]):

$$
f_{\text {teor }}=\frac{7}{\pi}\left[\frac{D_{\mathrm{o}}^{2}}{D_{\mathrm{D}}}\right] \sqrt{\frac{K_{\mathrm{p}}}{\rho\left(D^{2} L+14 D_{\mathrm{o}}^{2} L_{\mathrm{e}}\right)}},
$$

where $L_{\mathrm{e}}$ is the effective length calculated from [15]:

$$
L_{\mathrm{e}}=H+\frac{8}{3 \pi} D_{\mathrm{o}},
$$

where $H$ is the thickness of the pipe wall.

Experimentally evaluated resonance frequency is approximately $45 \mathrm{~Hz}$, this is in good agreement with the theoretical value $\left(f_{\text {theor }}=41.9 \mathrm{~Hz}\right)$.

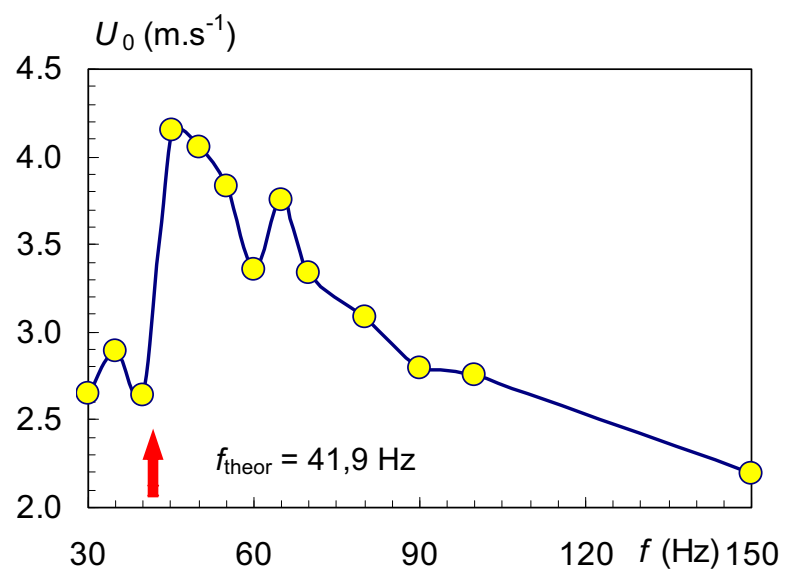

Fig. 3. Frequency characteristic.

The power characteristics were measured for five different frequencies $f=30,45,60,110$ and $160 \mathrm{~Hz}$. The
Pitot tube was again placed at $y / D_{\mathrm{o}}=6$ and $z=0 \mathrm{~mm}$. Characteristics were measured at $x=18,28$ and $38 \mathrm{~mm}$.

Figure 4 shows the power characteristics. Figure $4 \mathrm{~b}$ shows in detail the range of $0-0.2 \mathrm{~W}$. It can be seen that with decreasing power the velocity of SJ decreases too. For very low power input, the SJ was weak and probably near the formation criterion $[16,17]$.

(a)

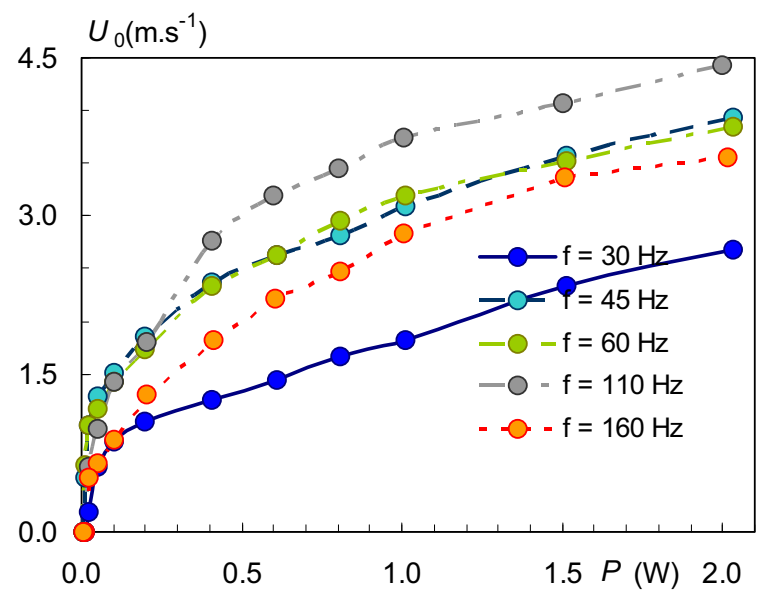

(b)

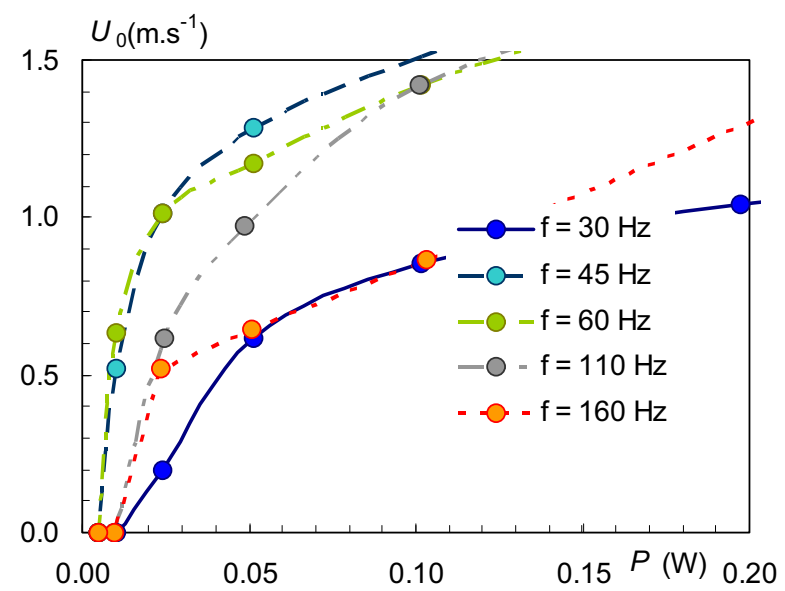

Fig. 4. Power characteristics a) whole characteristic, b) detail of the area with low values of the power.

\subsection{Flow visualization}

Figure 5a shows a visualization of the flow around the cylinder at the regime of the von Kármán vortex street; Fig. 5a and $5 \mathrm{~b}$ were made under continual and stroboscopic light, respectively. The natural vortex shedding frequency was $f=27.12 \mathrm{~Hz}$ and $S t=0.141$. In these cases, the SJs were off. 

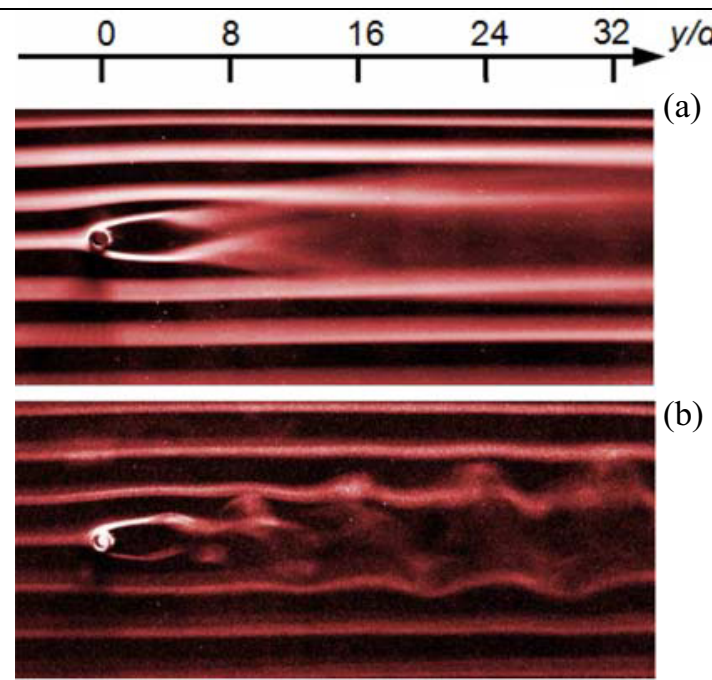

(b)

Fig. 5. Flow visualization of cylinder in cross-flow under (a) continual light, (b) stroboscopic light.

Visualization of the flow under the SJ control was made for five different $C_{\mathrm{f}}$, four different angles $\alpha=0$, 75,120 and $180^{\circ}$ and two values of $C_{\mu}=0.21$ and 1.02 .

Parameters of the flow visualization experiments for $C_{\mu}=0.21$ are summarized in Table 1 .

Table 1. Parameters of the visualization experiments for $C_{\mu}=0.21$.

\begin{tabular}{cccccc}
\hline Fig. & $\alpha\left(^{\circ}\right)$ & $P(\mathrm{~W})$ & $f_{\mathrm{sj}}(\mathrm{Hz})$ & $S t_{\mathrm{UO}}(1)$ & $C_{\mathrm{f}}(1)$ \\
\hline \hline 6a & 0 & & & & \\
$6 \mathrm{~b}$ & 75 & 0.080 & 27.2 & 0.041 & 1.00 \\
$6 \mathrm{c}$ & 120 & & & & \\
6d & 180 & & & & \\
\hline $7 \mathrm{a}$ & 0 & & & & \\
$7 \mathrm{~b}$ & 75 & 0.015 & 41.2 & 0.062 & 1.52 \\
$7 \mathrm{c}$ & 120 & & & & \\
$7 \mathrm{~d}$ & 180 & & & & \\
\hline $8 \mathrm{a}$ & 0 & & & & \\
$8 \mathrm{~b}$ & 75 & 0.015 & 53.9 & 0.081 & 1.99 \\
$8 \mathrm{c}$ & 120 & & & & \\
$8 \mathrm{~d}$ & 180 & & & & \\
\hline $9 \mathrm{a}$ & 0 & & & & \\
$9 \mathrm{~b}$ & 75 & 0.030 & 108.4 & 0.163 & 4.00 \\
$9 \mathrm{c}$ & 120 & & & & \\
$9 \mathrm{~d}$ & 180 & & & & \\
\hline $10 \mathrm{a}$ & 0 & & & & \\
$10 \mathrm{~b}$ & 75 & 0.080 & 162.4 & 0.244 & 5.99 \\
$10 \mathrm{c}$ & 120 & & & & \\
$10 \mathrm{~d}$ & 180 & & & & \\
\hline
\end{tabular}

Figures 6(a-d) show flow visualizations for $C_{\mathrm{f}}=1.00$. In all cases the separation point moved downstream under the flow control condition which caused reduction of the width of the wake. If the SJs were placed at the front stagnation point $\left(\alpha=0^{\circ}\right)$, small closed region was formed as a result of interaction between the SJs and the oncoming main flow as shown in Figure 6a.

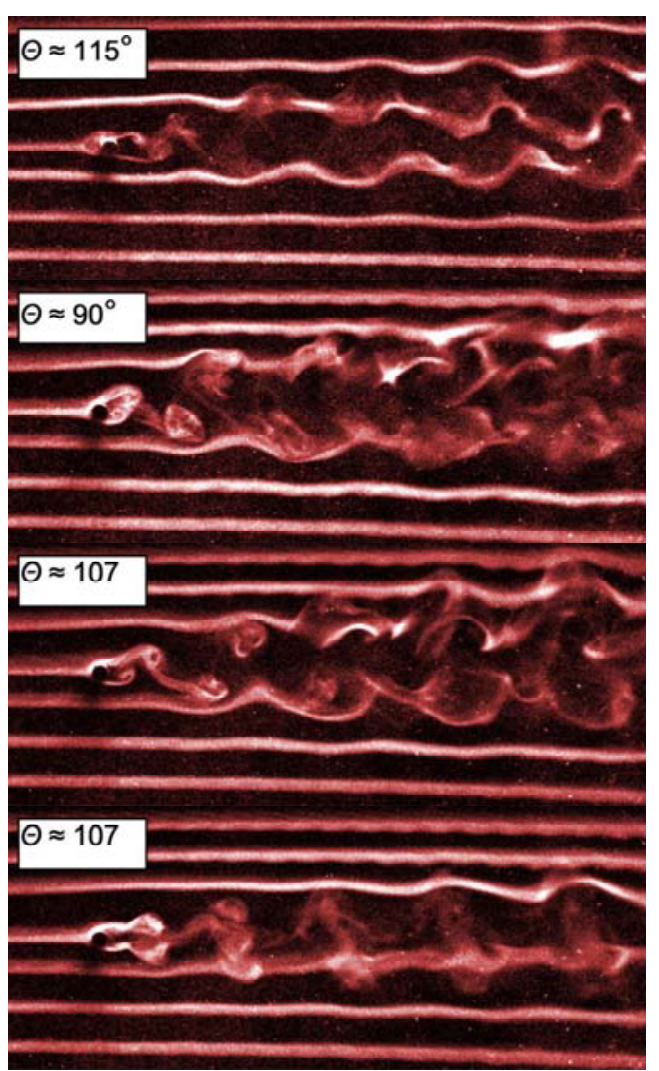

Fig. 6. Flow visualization for $C_{\mu}=0.21$ and $C_{\mathrm{f}}=1.00$ at (a) $\alpha=0^{\circ}$, (b) $\alpha=75^{\circ}$, (c) $\alpha=120^{\circ}$, (d) $\alpha=180^{\circ}$; (parameters are summarized in Table 1).

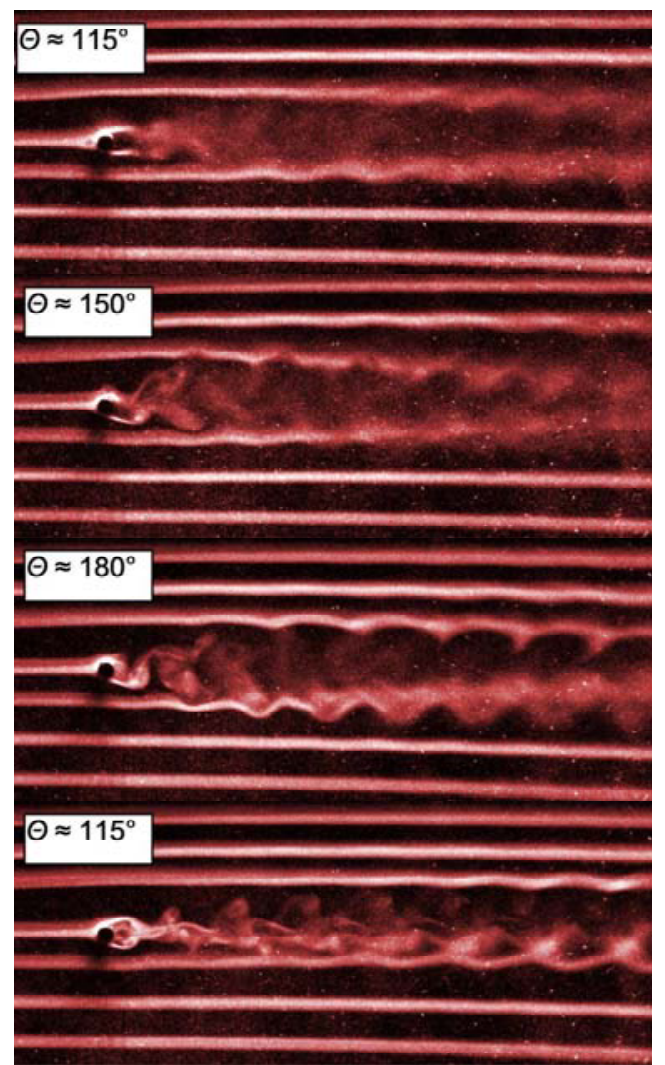

Fig. 7. Visualization for $C_{\mu}=0.21$ and $C_{\mathrm{f}}=1.52$ at (a) $\alpha=0^{\circ}$, (b) $\alpha=75^{\circ}$, (c) $\alpha=120^{\circ}$, (d) $\alpha=180^{\circ}$; (parameters - see Table 1). 


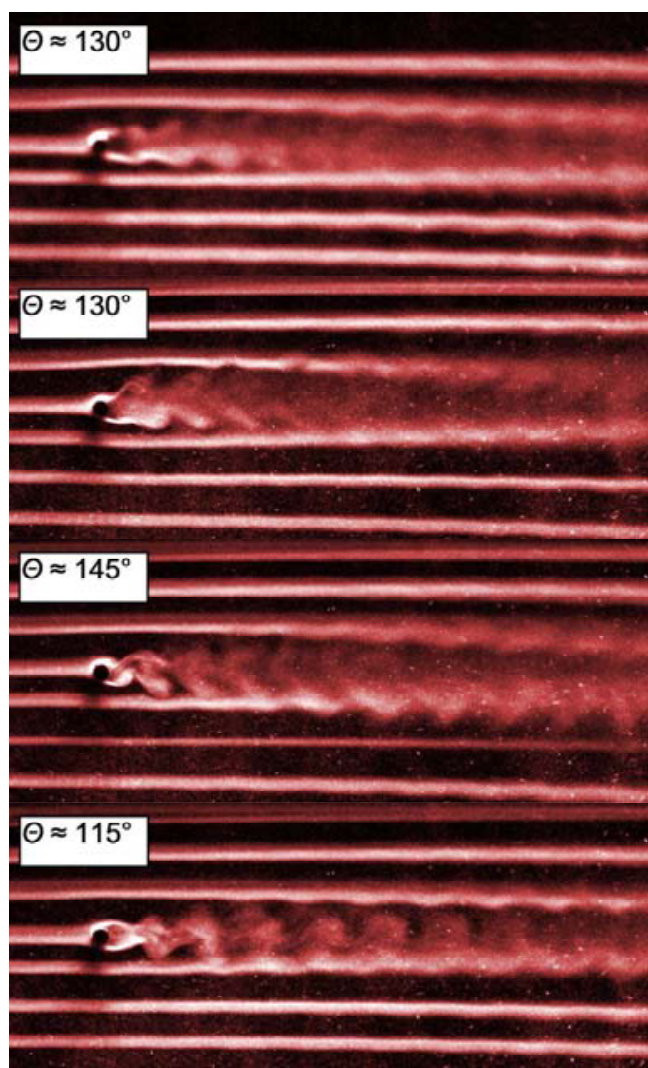

Fig. 8. Visualization for $C_{\mu}=0.21$ and $C_{\mathrm{f}}=1.99$ at (a) $\alpha=0^{\circ}$, (b) $\alpha=75^{\circ}$, (c) $\alpha=120^{\circ}$, (d) $\alpha=180^{\circ}$; (parameters - see Table 1 ).

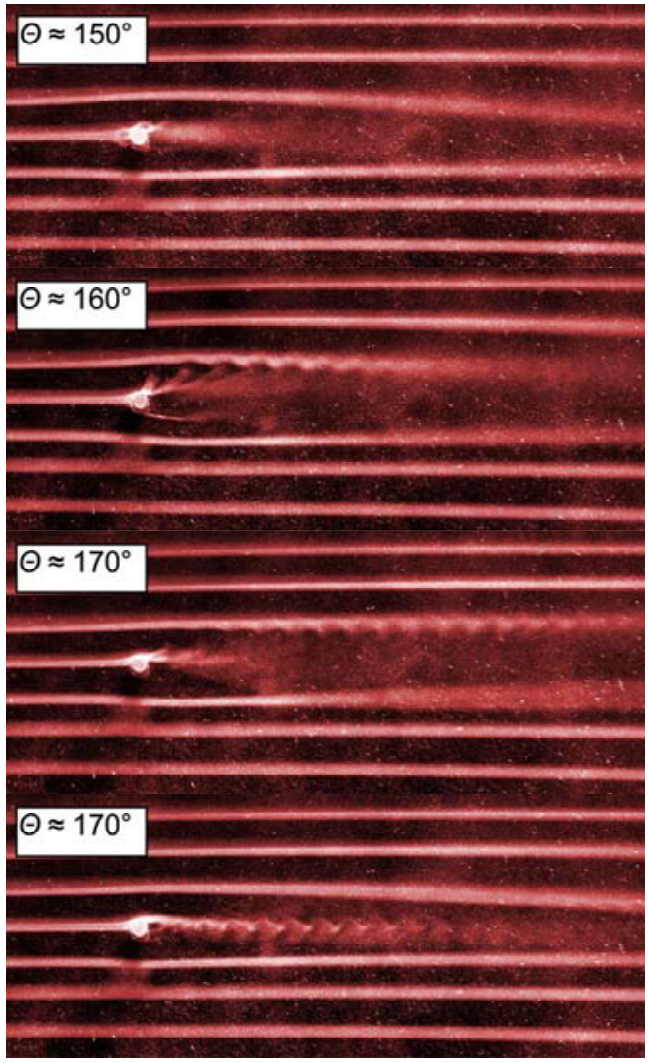

Fig. 9. Visualization for $C_{\mu}=0.21$ and $C_{\mathrm{f}}=4.00$ at (a) $\alpha=0^{\circ}$, (b) $\alpha=75^{\circ}$, (c) $\alpha=120^{\circ}$, (d) $\alpha=180^{\circ}$; (parameters - see Table 1).

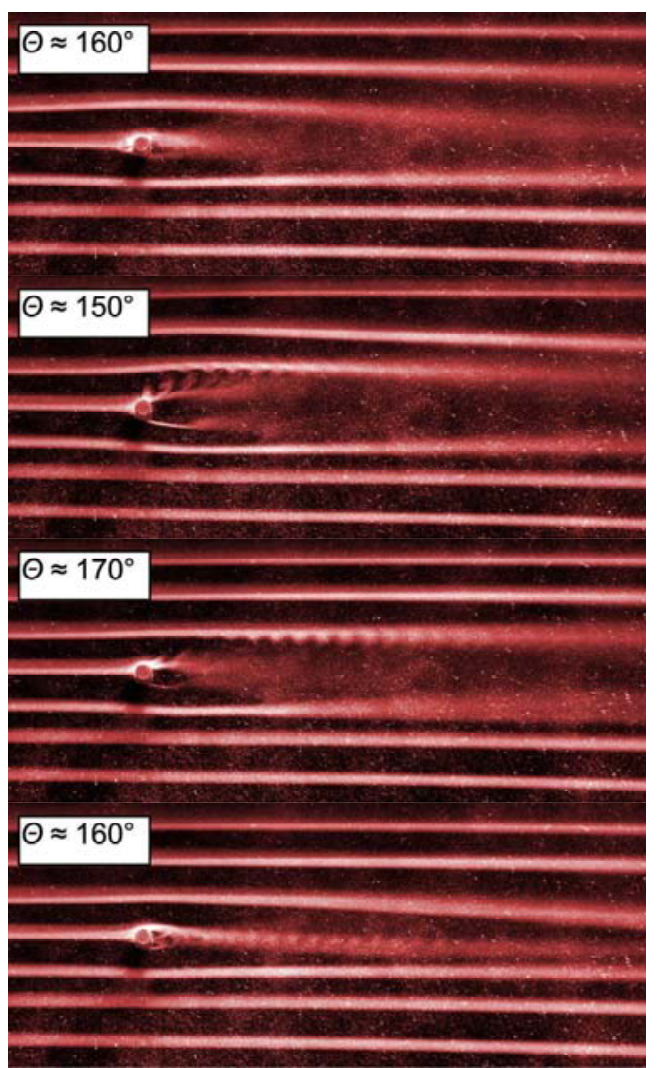

Fig. 10. Visualization for $C_{\mu}=0.21$ and $C_{\mathrm{f}}=5.99$ at (a) $\alpha=0^{\circ}$, (b) $\alpha=75^{\circ}$, (c) $\alpha=120^{\circ}$, (d) $\alpha=180^{\circ}$; (parameters - see Table 1).

This finding is in agreement with [7]. If the SJs were placed at the rear stagnation point $\left(\alpha=180^{\circ}\right)$ the wake behind the cylinder was symmetric.

Figures 7(a-d) show flow visualization for $C_{\mathrm{f}}=1.52$. With increasing frequency of the SJS, structures in the wake were refined and the separation point were moved more downstream.

With further increasing SJ frequency, a further refinement of the wake structure is visible as shown in Figures $8(\mathrm{a}-\mathrm{d})$ for $C_{\mathrm{f}}=1.99$. Apparently, this flow field pattern indicates the lock-on effect $[11,18,19]$. Closed region at the front stagnation point $\left(\alpha=0^{\circ}\right)$ is not so obvious, because the SJs were captured in the suction part of period. similarly as e.g. in [7].

Figures 9(a-d) show flow visualization for $C_{\mathrm{f}}=4.00$. In these cases, the von Kármán vortex street was completely suppressed. Structures in the wake were created by the SJs during the extrusion part of the period. If the SJs were placed at the stagnation points $(\alpha=0$ and $180^{\circ}$ ), width of the wake was approximately equal to the diameter of the cylinder.

Figures 10(a-d) show flow visualization of the flows with $C_{\mathrm{f}}=5.99$. This increase of frequency yielded no significant changes compared to the visualization for $C_{\mathrm{f}}=4.00$. Only structure in the wake was finer as a manifestation of the lock-on effect.

Another visualization experiments were made for stronger $\mathrm{SJ}$ at $C_{\mu}=1.02$. The parameters for this visualization experiments are sumarized in Table 2. 
Table 2. Parameters of the visualization experiments for $C_{\mu}=1.02$.

\begin{tabular}{cccccc}
\hline Fig. & $\alpha\left(^{\circ}\right)$ & $P(\mathrm{~W})$ & $f_{\mathrm{si}}(\mathrm{Hz})$ & $S t_{\mathrm{U} 0}(1)$ & $C_{\mathrm{f}}(1)$ \\
\hline \hline $11 \mathrm{a}$ & 0 & & & & \\
$11 \mathrm{~b}$ & 75 & & & & \\
$11 \mathrm{c}$ & 120 & 0.75 & 27.3 & 0.041 & 1.01 \\
$11 \mathrm{~d}$ & 180 & & & & \\
\hline $12 \mathrm{a}$ & 0 & & & & \\
$12 \mathrm{~b}$ & 75 & 0.12 & 41.0 & 0.062 & 1.51 \\
$12 \mathrm{c}$ & 120 & & & & \\
$12 \mathrm{~d}$ & 180 & & & & \\
\hline $13 \mathrm{a}$ & 0 & & & & \\
$13 \mathrm{~b}$ & 75 & & & & \\
$13 \mathrm{c}$ & 120 & 0.15 & 54.2 & 0.081 & \\
$13 \mathrm{~d}$ & 180 & & & & \\
\hline $14 \mathrm{a}$ & 0 & & & & \\
$14 \mathrm{~b}$ & 75 & \multirow{2}{*}{0.16} & 108.2 & 0.163 & \\
$14 \mathrm{c}$ & 120 & & & & \\
$14 \mathrm{~d}$ & 180 & & & & \\
\hline $15 \mathrm{a}$ & 0 & & & & \\
$15 \mathrm{~b}$ & 75 & \multirow{2}{*}{0.32} & 162.3 & & \\
$15 \mathrm{c}$ & 120 & & & & \\
$15 \mathrm{~d}$ & 180 & & & & \\
\hline
\end{tabular}

If the momentum coefficient was increased from $C_{\mu}=0.21$ to 1.02 , the interaction between the SJS and oncoming flow can no longer be considered a flow control, it is rather an interaction between two comparable flows.

Figures 11(a-d) show flow fields for $C_{\mathrm{f}}=1.01$. If the SJs were placed at the front stagnation point $\left(\alpha=0^{\circ}\right)$ the entire flow field in the observed area was disturbed. Because of higher $C_{\mu}$, the separation angle $\theta$ was almost same as the angle $\alpha$. If the SJs were placed at the rear stagnation point $\left(\alpha=180^{\circ}\right)$, flow separation was not observed either on the upper, or on the bottom sides of the cylinder.

Figures 12(a-d) show flow visualization for $C_{\mathrm{f}}=1.51$. The increase of the SJ frequency reduced the disturbed region in front of the cylinder.

Figures 13(a-d) show interactions between the SJs and the oncoming flow for $C_{\mathrm{f}}=2.00$. Open envelope was observed if the SJs were placed at the front stagnation point $\left(\alpha=0^{\circ}\right)$. This is in good agreement with [7]. Figures 14 and 15 show visualization for $C_{\mathrm{f}}=3.99$ and $C_{\mathrm{f}}=5.98$, respectively. Structures in the wake were more refined because of the lock-on effect. As shown in Figures 12-15, the separation point on the surface of the cylinder was moved downstream.

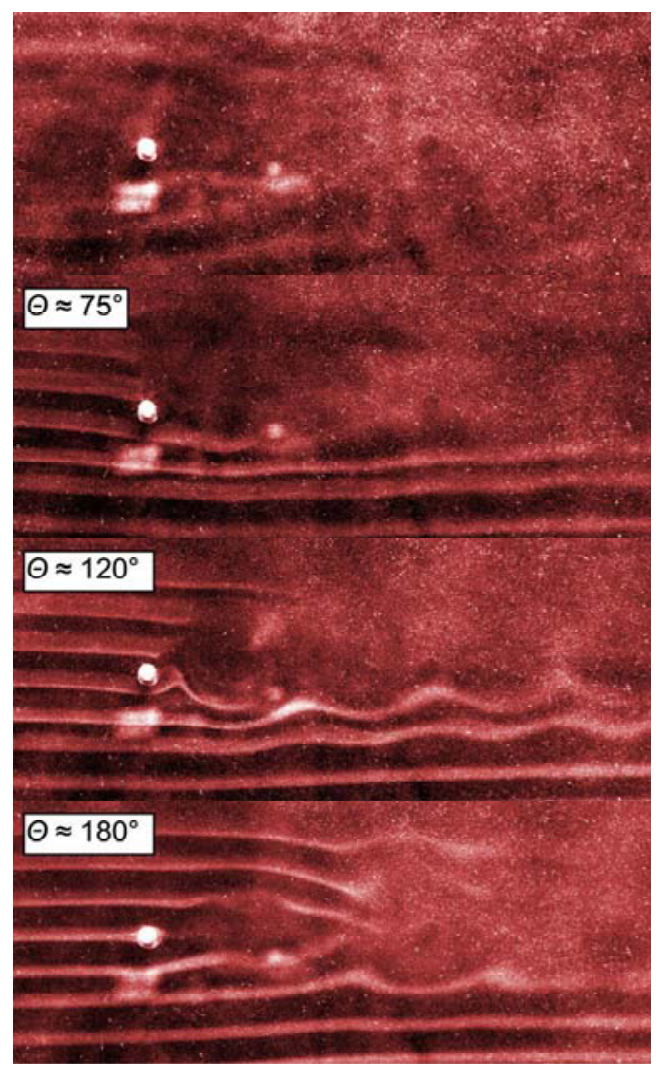

Fig. 11. Visualization for $C_{\mu}=1.02$ and $C_{\mathrm{f}}=1.01$ at (a) $\alpha=0^{\circ}$, (b) $\alpha=75^{\circ}$, (c) $\alpha=120^{\circ}$, (d) $\alpha=180^{\circ}$; (parameters - see Table 2).

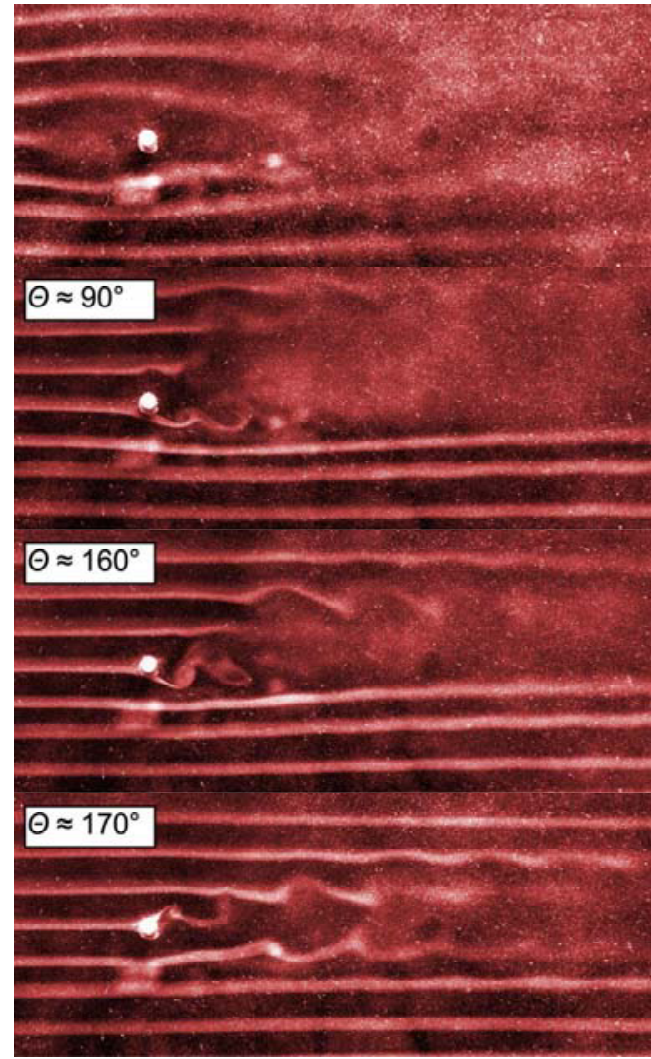

Fig. 12. Visualization for $C_{\mu}=1.02$ and $C_{\mathrm{f}}=1.51$ at (a) $\alpha=0^{\circ}$, (b) $\alpha=75^{\circ}$, (c) $\alpha=120^{\circ}$, (d) $\alpha=180^{\circ}$; (parameters - see Table 2). 


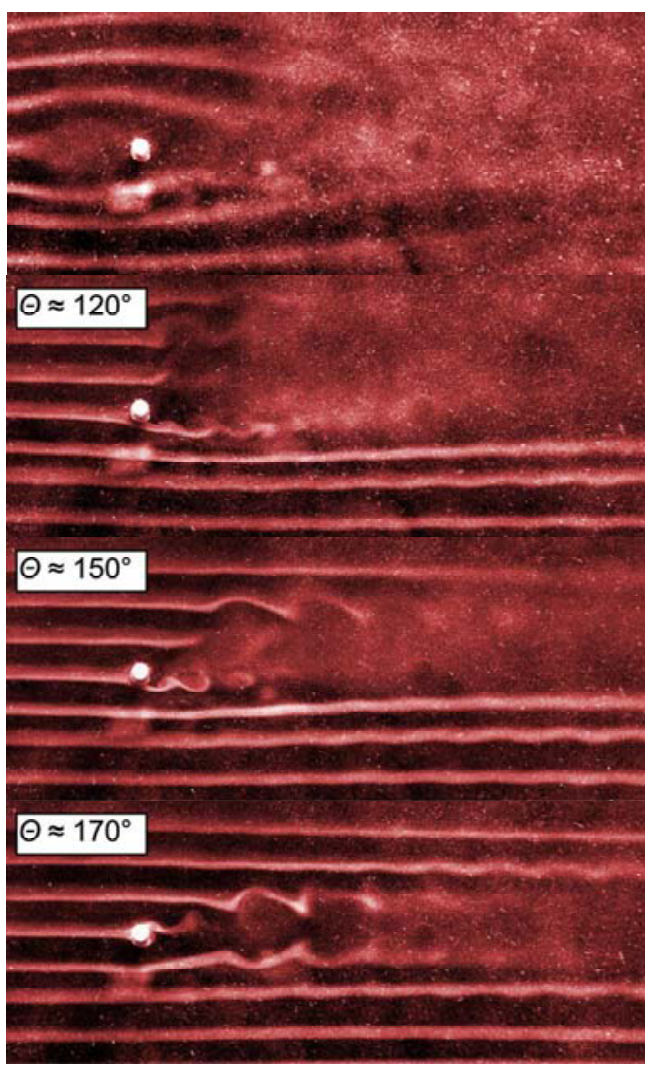

Fig. 13. Visualization for $C_{\mu}=1.02$ and $C_{\mathrm{f}}=2.00$ at (a) $\alpha=0^{\circ}$, (b) $\alpha=75^{\circ}$, (c) $\alpha=120^{\circ}$, (d) $\alpha=180^{\circ}$; (parameters - see Table 2).

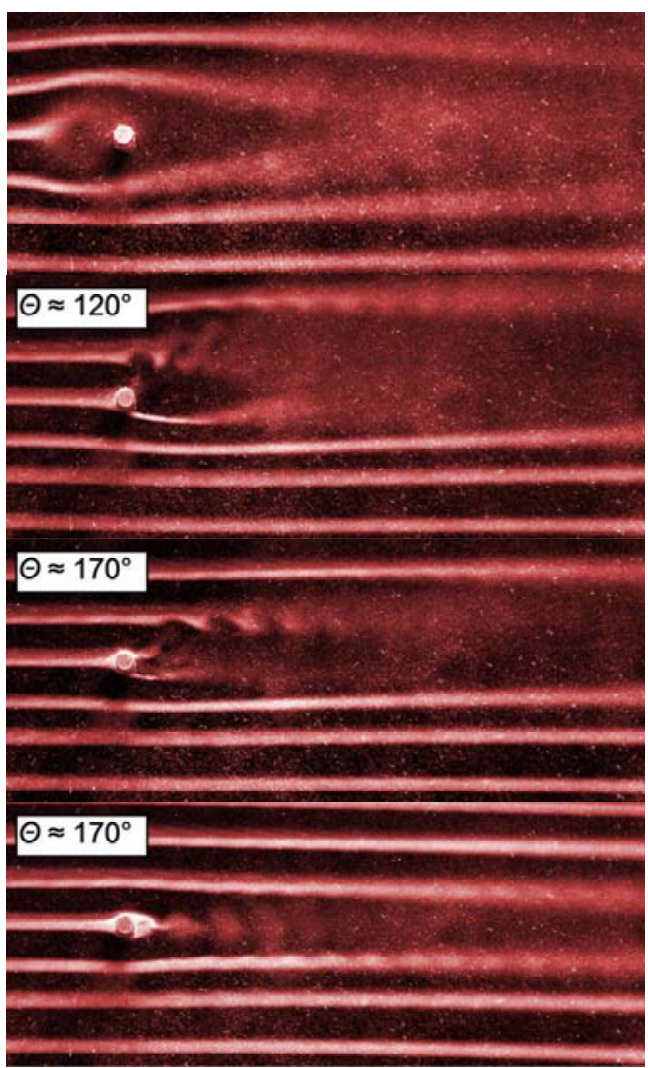

Fig. 14. Visualization for $C_{\mu}=1.02$ and $C_{\mathrm{f}}=3.99$ at (a) $\alpha=0^{\circ}$, (b) $\alpha=75^{\circ}$, (c) $\alpha=120^{\circ}$, (d) $\alpha=180^{\circ}$;

(parameters - see Table 2).

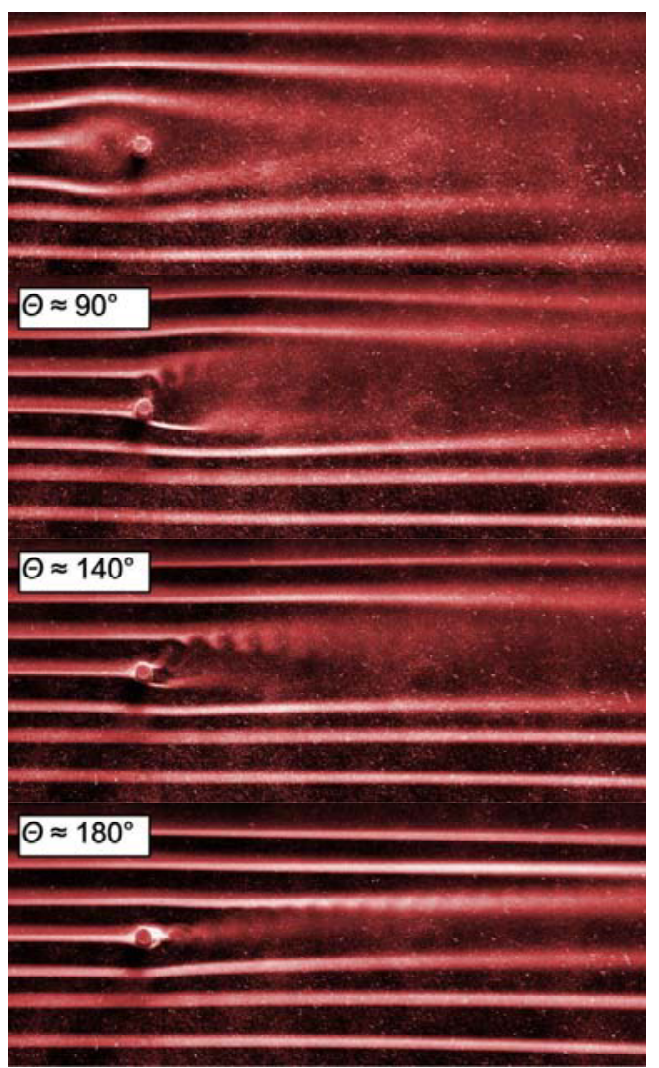

Fig. 15. Visualization for $C_{\mu}=1.02$ and $C_{\mathrm{f}}=5.98$ at (a) $\alpha=0^{\circ}$, (b) $\alpha=75^{\circ}$, (c) $\alpha=120^{\circ}$, (d) $\alpha=180^{\circ}$; (parameters - see Table 2).

\section{Conclusions}

The aim of this work was an experimental investigation of the flow field around the circular cylinder under the active flow control. The row of the 14 synthetic jets issuing from the cylinder surface was used for this purpose.

Firstly, the frequency and power characteristics were experimentally evaluated. Then a flow visualization study in the wind tunnel was performed. The most significant effects of the SJ control on the flow field are briefly summarized in the following points:

- An increase of the frequency ratio $C_{\mathrm{f}}$ influenced vortex structures in the wake. More specifically, the large vortex structures were refined with increasing $C_{\mathrm{f}}$.

- The lock-on effect was indicated for majority of experiments at $C_{\mathrm{f}} \geq 1.5$.

- The separation point was moved downstream. When the momentum coefficient was $C_{\mu}=0.21$, the separation angles were $\theta \approx 130-160^{\circ}$. For $C_{\mu}=1.02$ the separation angles were $\theta \approx 90-160^{\circ}$. If the SJs were placed at the rear stagnation point $\left(\alpha=180^{\circ}\right)$, no separation was observed in some cases, see Figures $11 \mathrm{~d}$ and $15 \mathrm{~d}$.

- For $C_{\mu}=0.21$, the flow control always reduces the wake width. The same observation was made for $C_{\mu}=1.02$ except the cases of the SJS at the front stagnation point $\left(\alpha=0^{\circ}\right)$. 
- If the SJs were placed at the front or rear stagnation points $\left(\alpha=0\right.$ or $\left.180^{\circ}\right)$, the wake was symmetric in majority cases as shown in Figures $6 d, 7(a, d), 9(a, d)$ $10 \mathrm{~d}, 11 \mathrm{~d}, 14 \mathrm{~d}$ and $15 \mathrm{~d}$.

\section{Acknowledgments}

We gratefully acknowledge the support of the GACR Czech Science Foundation (projects no. 14-08888S and 16-16596S) and the institutional support RVO: 61388998 .

\section{References}

1. M. Gad-El-Hak, Flow Control (Cambridge, University Press, New York, 2000)

2. B.L. Smith, A. Glezer, Phys. Fluids 10, pp. 22812297 (1998)

3. J. E. Cater, J. Soria, J. Fluid Mech. 472, pp. 167 200 (2002)

4. L-H. Feng, J-J. Wang, Proceedings of 16th International Symposium on Flow Vizualization, June 24-28, Okinawa, Japan (2014)

5. P-F. Zhang, J-J. Wang, L-H. Feng, Sci. ChinaTechnol. Sci. 51, pp. 1315 -1344 (2008)

6. M. Amitay, A. Honohan, M. Trautman, A. Glezer, AIAA Paper 97-2004 (1997)

7. J-J. Wang, L-H. Feng, Ch-J. Xu, Sci. ChinaTechnol. Sci. 50, pp. $550-559$ (2007)

8. L-H. Feng, J-J. Wang, Eur. J. Mech. B-Fluids 43, pp. $14-32(2014)$

9. L-H. Feng, J-J. Wang, Ch. Pan, J. Fluids Struct. 26, pp. $900-917$ (2010)

10. L-H. Feng, J-J. Wang, Exp. Fluids 53, pp. $467-480$ (2012)

11. L-Q. Ma, L-H. Feng, Sci. China-Technol. Sci. 56, pp. $158-170$ (2013)

12. J. Tensi, I. Boué, F. Paillé, G. Dury, J. Vis. 5, pp. 37 -44 (2002)

13. M. M. Zdravkovich, Flow around a Circular Cylinders (Oxford University Press, 1997)

14. Z. Broučková, Z. Trávníček, J. Vis. 18, pp. 581-593 (2015)

15. Z. Škvor, Akustika a elektroakustika (Acoustic and Electro-acoustic, Praha: Academia 2001, in Czech)

16. R. Holman, Y. Utturkar, R. Mittal, B.L. Smith, L. Cattafesta, AIAA J. 43, pp. 2110 - 2116 (2005)

17. Z. Trávníček, Z. Broučková, J. Kordík, AIAA J. 50, pp. $2012-2017$ (2012)

18. L. H. Feng, J. J. Wang, J. Fluid Mech. 662, pp. 232 -259 (2010)

19. W. Kim, J. Y. Yoo, J. Sung, Phys. Fluids 18, pp. 1 20 (2006)

20. E. Flídr, Kruhový válec v homogenním proudovém poli s vlivem aktivního ř́zení (Circular Cylinder in Homogeneous Flow Field under an Active Control Influence, Master Thesis, Faculty of Mechanical Engineering, CTU in Prague, 2016, in Czech). 